\title{
Tratamientos profilácticos para desinfectar la superficie de huevos del pargo flamenco Lutjanus guttatus
}

Prophylactic treatments for surface disinfection of spotted rose snapper Lutjanus guttatus eggs

\author{
Leonardo Ibarra-Castro ${ }^{1}$, Cynthia R. Lizarraga-Osuna ${ }^{2}$, \\ Bruno Gómez-Gil ${ }^{1}$ y Luis Alvarez-Lajonchère ${ }^{3}$
}

\begin{abstract}
${ }^{1}$ Centro de Investigación en Alimentación y Desarrollo (CIAD), A. C., Unidad Mazatlán, Avenida Sábalo Cerritos S/N, Mazatlán, C.P. 82010, A.P. 711, Sinaloa, México

${ }^{2}$ Universidad Autónoma de Sinaloa, Facultad de Ciencias del Mar, PaseoClaussen s/n P.O Box 1132, Mazatlán Sinaloa, 82000, Sinaloa, México

${ }^{3}$ Grupo Piscimar, Calle 41 No. 886, Nuevo Vedado, Plaza, La Habana, C.P. 10600, Cuba. alajonchere@gmail.com
\end{abstract}

\begin{abstract}
For incubation of eggs, prophylactic treatments are applied to minimize negative effects of microorganisms on eggs and larvae. The effects of treatments with formaldehyde, acriflavine and glutaraldehyde to spotted rose snapper Lutjanus guttatus eggs, were studied. Reduction of colony forming units (CFU), the percentages of hatching, live larvae, live normal larvae and larval size, were studied. Best treatment with significant reduction of CFU, but without observable effects on the eggs with controls was with acriflavine at 5 ppm.
\end{abstract}

Palabras clave: Incubation, egg surface prevention procedures, marine larviculture

\section{INTRODUCCIÓN}

El pargo flamenco, Lutjanus guttatus (Steindachner, 1869) se cultiva experimental y comercialmente en América Latina (Avilés-Quevedo et al. 2008), por lo cual se han realizado diversos estudios sobre la producción masiva de juveniles (Alvarez-Lajonchère et al. en prensa ${ }^{1}$ ). Debido a las altas mortalidades en larvicultura, se requieren procedimientos para elevar la supervivencia larval de una producción masiva de juveniles.

La incubación de huevos y la cría de larvas están íntimamente relacionados con la maduración y el desove y muchos de los efectos que se aprecian en la incubación o en la etapa de larvicultura tienen su origen en la etapa de los reproductores, incluyendo la contaminación por microorganismos (Grotmol et al. 2003). Además, la incubación de huevos generalmente se realiza en alta densidad, lo cual favorece el crecimiento microbiano (Douillet \& Holt 1994, Hansen \& Falk-Petersen 2001).

Se ha demostrado que la contaminación bacteriana durante la incubación puede afectar los porcentajes de eclosión, especialmente en especies de incubación prolongada (Hansen \& Falk-Petersen 2001); sin embargo, los efectos más significativos se manifiestan en la etapa de larvicultura (Douillet \& Holt 1994, Verner-Jeffreys et al. 2007). La introducción de huevos o larvas en los tanques de larvicultura puede propiciar la contaminación vertical con microorganismos patógenos y por ser un período prolongado, los daños pueden ser significativos (Douillet \& Holt 1994).

Los tratamientos profilácticos de huevos pueden minimizar la introducción de microorganismos patógenos por disminuir la carga microbiana en su superficie (Douillet $\&$ Holt 1994, Moretti et al. 1999). Hace algunos años, algunos tratamientos profilácticos se realizaban con antibióticos (Moretti et al., 1999), pero debido al peligro de propiciar la resistencia de los microorganismos, recientemente se basan en baños con agentes químicos a concentraciones y tiempo específicos, como compuestos del yodo (Verner-Jeffreys et al. 2007, Stuart et al. 2010), formalina (Holt 2005, Stuart et al. 2010), peróxido de hidrógeno (Douillet \& Holt 1994, VernerJeffreys et al. 2007), acriflavina (Tiensongrusmee et al. 1989), glutaraldehido (Salvesen \& Vadstein 1995, Katharios et al. 2007) y ozono (Grotmol et al. 2003, Ben-Atia et al. 2007). Muchos de estos desinfectantes han sido utilizados también como tratamientos terapéuticos (Gieseker et al. 2006).

\footnotetext{
${ }^{1}$ Alvarez-Lajonchère L, MI Abdo de la Parra, LE Rodríguez-lbarra, G Velasco-Blanco, A Puello-Cruz, B González-Rodríguez, N GarcíaAguilar \& A Ibarra-Soto. Pilot-scale larval rearing of spotted rose snapper (Lutjanus guttatus) at Mazatlan, Mexico. Journal of the World Aquaculture Society. [en prensa].
} 
La mayoría de los estudios de tratamientos profilácticos para peces marinos se han realizado con especies subtropicales y de aguas frías, con períodos de incubación de varios días y con presencia de microorganismos diferentes a los que se pueden encontrar en aguas tropicales. El objetivo del presente estudio fue determinar los efectos de varios tratamientos profilácticos de desinfección de huevos del pargo flamenco para obtener larvas viables con la menor carga posible de microorganismos potencialmente patógenos y las menores afectaciones, dada la escasa información sobre especies tropicales, así como el hecho de que la efectividad de los tratamientos depende de las características las especies (Douillet \& Holt 1994, Grotmol et al. 2003, Ben-Atia et al. 2007).

\section{Materiales Y MÉTODOS}

En este estudio se aplicó el enfoque toxicológico basado en la determinación de las concentraciones con efectos no observables (CENO) para cada germicida, definidas como aquellas sin efectos negativos comparadas con un control (Douillet \& Holt 1994) y a la vez, que logren reducciones efectivas de la carga bacteriana de los huevos, definida como menos de 10 unidades formadoras de colonias (UFC) huevo-1 (Verner-Jeffreys et al. 2007).

Los huevos fertilizados fueron obtenidos por desoves naturales de un grupo de reproductores de pargo flamenco mantenidos en cautiverio en la planta piloto del Centro de Investigación en Alimentación y Desarrollo A. C., CIAD-Mazatlán, cuyos métodos de cría fueron descritos por Ibarra-Castro \& Alvarez-Lajonchère (2011). Los huevos fueron extraídos de los colectores en el rebozo de los tanques de desove, 12-14 h después de la fertilización, en cantidades de más de un millón de huevos diariamente con alta calidad (más de un $90 \%$ de huevos flotantes con una viabilidad del $95 \%$ y un porcentaje de fertilización de más del 88\%). El número de huevos flotantes, fue estimado volumétricamente según lo descrito por Ibarra-Castro \& Alvarez-Lajonchère (2011) y posteriormente los flotantes fueron lavados con agua filtrada hasta $1 \mu \mathrm{m}$ de retención absoluta e irradiada en una lámpara UV de flujo continuo $\left(60 \mathrm{~mJ} \mathrm{~cm}^{-2}\right)$ e introducidos en un recipiente de $500 \mathrm{~mL}$ para usarlos en cada experimento. La salinidad en los tratamientos experimentales y las incubaciones se mantuvo entre $35-36$ ups y la temperatura entre $28-30^{\circ} \mathrm{C}$.

Se evaluaron tres sustancias desinfectantes de calidad técnica (Sigma, Toluca, México) y cada una se probó a tres concentraciones: formalina $(10,25$ y $50 \mathrm{ppm})$, acriflavina $(2,5,5,0$ y $7,5 \mathrm{ppm})$ y glutaraldehido $(100,200$ y $400 \mathrm{ppm})$. Cada tratamiento tuvo 3 réplicas y cada uno se repitió en 3 ocasiones. Los tratamientos se aplicaron en forma de baño en los que se sumergieron los huevos $(1 \mathrm{~h}$ para la formalina, 1 min para la acriflavina y $10 \mathrm{~min}$ para el glutaraldehido) en vasos de precipitado de $250 \mathrm{~mL}$ con $150 \mathrm{~mL}$ de las soluciones y un control por triplicado, todos con aireación constante. Las muestras de huevos flotantes se tomaron con una pipeta de precisión Finnpipette ${ }^{\circledR}$ R, $10 \mathrm{~mL}$ para la acriflavina y el glutaraldehido y $5 \mathrm{~mL}$ para la formalina para lograr la proporción deseada de densidad de huevos y solución del baño en este caso.

Para las determinaciones de las UFC, se utilizaron dos medios de cultivo, el Zobell o agar marino (AM) para bacterias heterótrofas marinas y el agar tiosulfato-citratosales biliares-sacarosa (TCBS) para vibrios. El medio Zobell se preparó con $0,70 \mathrm{~g}$ de extracto de levadura, 3,50 g de bactopeptona $0,70 \mathrm{~mL}$ de $\mathrm{FeCl}$ solución acuosa al $15 \%, 15 \mathrm{~g}$ agar bacteriológico, $750 \mathrm{~mL}$ de agua de mar y $250 \mathrm{~mL}$ de agua; se ajustó a un $\mathrm{pH}$ de 7,5. Los conteos de las UFC se realizaron en un contador Darkfield Québec $\AA$, después de incubar las placas inoculadas a $30^{\circ} \mathrm{C}$ por $24 \mathrm{~h}$; las cajas Petri con medio Zobell se incubaron nuevamente con conteos a las $48 \mathrm{~h}$ y $72 \mathrm{~h}$.

Después de aplicado cada tratamiento, las muestras se filtraron por un tamiz de $500 \mu \mathrm{m}$, se enjuagaron con agua de mar tratada y se tomaron $100 \mu \mathrm{L}$ de huevos $(262$ huevos como promedio de 48 muestras de $100 \mu \mathrm{L}$ de huevos) y se sembraron en vasos de precipitado de 250 $\mathrm{mL}$ a un volumen de $150 \mathrm{~mL}$ con agua de mar tratada. De cada réplica se tomó $100 \mu \mathrm{L}$ de huevos, se maceraron en un tubo de ensayo eppendorf de $1 \mathrm{~mL}$ y se aforó a $1 \mathrm{~mL}$ de solución salina estéril. Se inocularon $100 \mu \mathrm{L}$ por duplicado en cada medio de cultivo de bacterias. En cada caso se evaluó el nivel de reducción bacteriana, medido por la disminución de las UFC huevo-1.

A las $24 \mathrm{~h}$ de iniciar la incubación, se evaluaron los siguientes índices:

Porcentaje de eclosión $=\mathrm{LV}+\mathrm{LM} \times 100 / \mathrm{LV}+\mathrm{LM}+\mathrm{H}$

Porcentaje de supervivencia $=$ LV x $100 / L V+L M$

Porcentaje de larvas normales $=\mathrm{LN}$ x $100 / \mathrm{LV}$

donde $\mathrm{LV}=$ larvas vivas; $\mathrm{LN}=$ larvas normales; $\mathrm{LM}=$ larvas muertas; $\mathrm{H}=$ huevos.

Se midió el largo total de 25 larvas recién eclosionadas por réplica en cada una de las tres repeticiones (225 por tratamiento), después de anestesiadas con una gota de 
2-fenoxietanol (a $150 \mathrm{ppm}$ ), con un microscopio estereoscópico con micrómetro ocular y $25 \mu \mathrm{m}$ de precisión.

Los resultados se presentan por sus valores medios \pm la desviación estándar para cada una de las variables determinadas y para el caso de longitud de larvas se presenta como el promedio \pm el error estándar de la media. Los porcentajes fueron transformados a arcoseno. La homogeneidad de las varianzas y distribución normal de los datos fueron analizados siguiendo los procedimientos estadísticos del software. Los datos que cumplieron las pruebas paramétricas se analizaron por ANDEVA de una vía y cuando este fue significativo se continuó con una prueba de comparación múltiple (prueba de Tukey). Los datos no paramétricos fueron analizados por la prueba de Kruskal-Wallis de rangos y cuando se detectaron diferencias significativas, se continuó con la prueba de comparación múltiple de Dunn. Las diferencias estadísticas significativas fueron reportadas cuando $P<$ 0,05 . Los análisis estadísticos fueron realizados en el software SigmaStat 3.5 para Windows (SYSTAT Software, Inc., Point Richmond, CA, USA).

\section{RESULTADOS Y DISCUSIÓN}

En todos los tratamientos, las UFC fueron reducidas significativamente a menos de $10 \mathrm{UFC} \mathrm{huevo}^{-1}$ respecto a las UFC huevo ${ }^{-1}$ del control, nivel de desinfección efectiva (Verner-Jeffreys et al. 2007) (Kruskal-Wallis, $\mathrm{H}=21,78$ a $31,09, P<0,001$ ) (Fig. 1); sin embargo, sólo con formalina y glutaraldehido se logró niveles de esterilidad $(<1$ UFC huevo-1) en la superficie de los huevos. No hubo diferencias significativas entre los tratamientos (ANDEVA, $\mathrm{F}_{8,80}=1,80, P>0,05$ ).

Verner-Jeffreys et al. (2007) reportaron niveles bacterianos más elevados en algunos lotes de huevos no tratados respecto al presente estudio; sin embargo, el lavado con agua tratada disminuyó su carga bacteriana, como los reportes de Dehasque et al. (1991) y Ben-Atia et al. (2007). La presencia de una carga bacteriana notablemente alta $\left(>10^{4}-10^{6} \mathrm{UFC}\right.$ huevo $\left.{ }^{-1}\right)$ puede ser una indicación de baja calidad de los huevos (Moretti et al. 1999).
Figura 1. Resultados de las unidades formadoras de colonias huevo-1 sembrados en los medios Agar Marino y TCBS a las $72 \mathrm{~h}$ y a las $24 \mathrm{~h}$ respectivamente con los tratamientos con: A) formalina, B) acriflavina y C) glutaraldehido. Cada barra representa el promedio \pm error estándar de la media de las tres réplicas con las tres repeticiones $(n=9)$ en cada tratamiento con huevos del pargo flamenco Lutjanus guttatus. Las letras minúsculas diferentes indican diferencias significativas (ANDEVA, $P<0,05$ ) respecto a los controles / Results on colony forming unites (CFU) egg ${ }^{-1}$ stocked on TCBC and Marine Agar media at $72 \mathrm{~h}$ and $24 \mathrm{~h}$ respectively, with prophylactic treatments with: $A$ ) formalin, B) acriflavine and C) glutaraldehyde. Each bar represents the mean \pm standard error of the mean of three replica repeated three times $(n=9)$ in each experiment with spotted red snapper Lutjanus guttatus, eggs. Different lower case letters indicate significant differences compared with controls (ANOVA, $P<0.05$ )
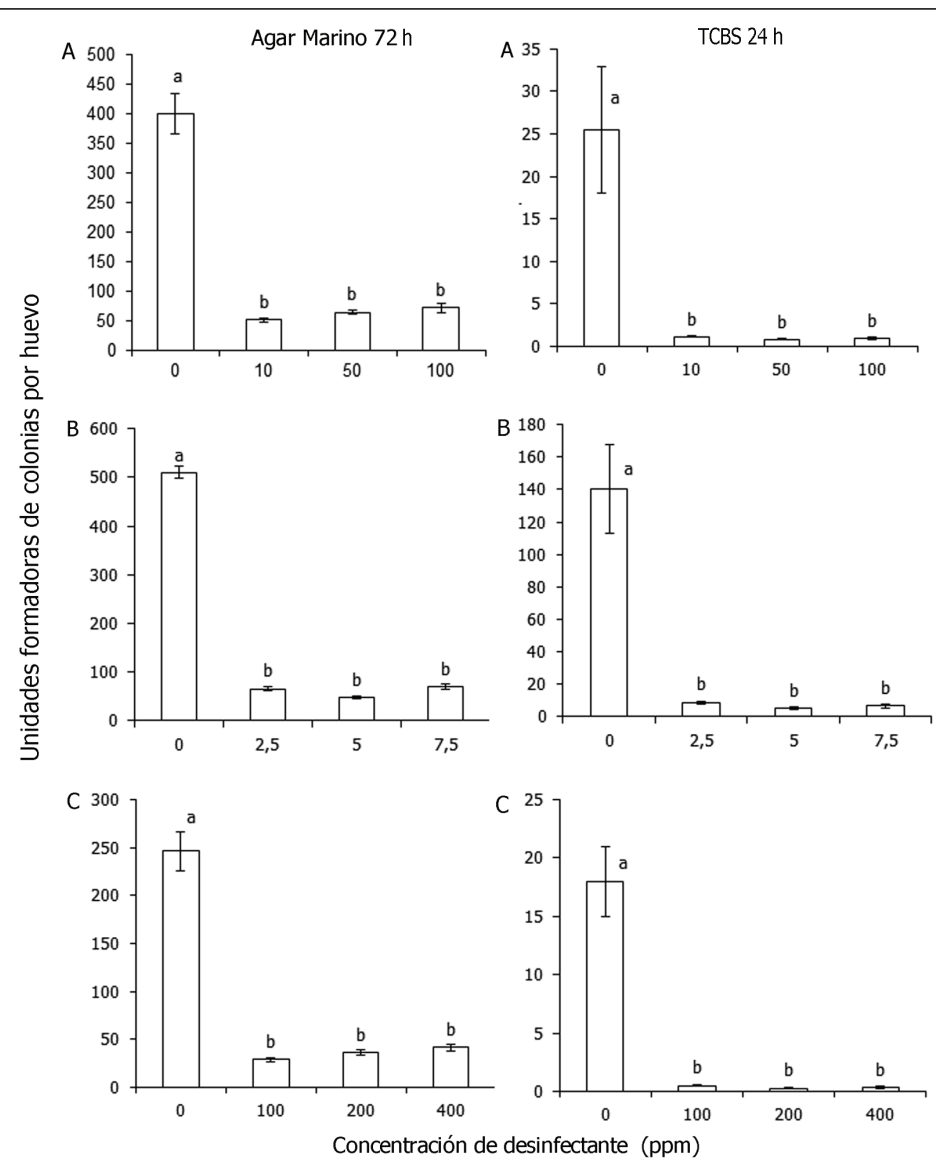
Los porcentajes de eclosión con formalina presentaron diferencias significativas con el control $(91,4 \%)$ (ANDEVA, $\mathrm{F}_{3,32}=103,14, P<0,001$ ), excepto a la concentración de $10 \mathrm{ppm}$ con $89,0 \%$ (Tukey, $Q_{3,32}=0,866$, $P>0,05)$ (Fig. 2). Con acriflavina los porcentajes de larvas vivas también presentaron diferencias respecto al control (94,0\%) (Kruskal-Wallis, $\mathrm{H}_{3,32}=26,700, P \leq 0,001$ ), excepto a 5 ppm (87,7\%) (Dunn, $Q_{3,32}=1,678, P>0,05$ ) (Fig. 2). Con glutaraldehido hubo diferencias con el control (ANDEVA, $\left.\mathrm{F}_{3,32}=17,452, P<0,001\right)$, debido a la concentración de $400 \mathrm{ppm}$, que fue también significativamente diferente del resto (Tukey, $Q_{3,32}=0,050$, $P<0,05)$. El porcentaje de eclosión con formalina a 10 ppm $(89,0 \%)$ no fue mayor que con acriflavina a 5 ppm (87,6\%), (Tukey, $\left.\mathrm{Q}_{28,8}=0,4202, P>0,05\right)$.

Con formalina, los porcentajes de larvas vivas presentaron diferencias respecto al control $(42,8 \%)$ (ANDEVA, $\mathrm{F}_{3,32}=154,11, P<0,001$ ), excepto a $10 \mathrm{ppm}$ $(39,4 \%)$ (Tukey, $\left.\mathrm{Q}_{3,32}=0,294, P>0,05\right)$ (Fig. 2). Con acriflavina los resultados también mostraron diferencias con el control (52,7 \%) (Kruskal-Wallis, $\mathrm{H}_{3,32}=28,776, P \leq$ 0,001 ), excepto a 5 ppm con $47,6 \%$ (Dunn, $\mathrm{Q}_{3,32}=1,700, P$ $>0,05)$. Con glutaraldehido también hubo diferencias de los tratamientos con el control (ANDEVA, $\mathrm{F}_{3,32}=11,608$, $P<0,001$ ), por la concentración de $400 \mathrm{ppm}$ (Tukey, $\mathrm{Q}_{3,32}$ $=2,92, P<0,001)$ (Fig. 2). La comparación de las supervivencias de las larvas vivas entre los tratamientos fue significativa (ANDEVA, $\mathrm{F}_{8,80}=34,04, P<0,0001$ ) y los mejores resultados fueron con acriflavina $5 \mathrm{ppm}(47,6 \%)$, seguidos por el de formalina a $10 \mathrm{ppm}(39,4 \%)$, pero no hubo una diferencia significativa entre ellos (Tukey, $\mathrm{Q}_{28}$. $\left.{ }_{8}=3,951, P>0,05\right)$.

Los porcentajes de larvas normales en los tratamientos con formalina presentaron diferencias significativas con el control (60,3\%) (ANDEVA, $\mathrm{F}_{3,32}=5,128, P=0,001$ ), excepto a $10 \mathrm{ppm}(54,4 \%)$ (Tukey, $\left.\mathrm{Q}_{3,32}=0,294 P \geq 0,05\right)$ (Fig. 2). Los tratamientos con acriflavina no fueron significativamente diferentes del control $(56,9 \%)$ (ANDEVA, $\mathrm{F}_{3,32}=2,183, P=0,109$ ) y el mejor fue con 5 ppm (67,3\%) (Fig. 2). Con glutaraldehido los porcentajes de larvas normales presentaron diferencias significativas respecto al control (ANDEVA, $\mathrm{F}_{3,32}=3,010, P=0,045$ ), debidas a la concentración de $200 \mathrm{ppm}$ (Tukey, $\mathrm{Q}_{3,32}=$ $0,050, P<0,05)$ (Fig. 2). Entre los tratamientos, el porcentaje de larvas normales con acriflavina a $5 \mathrm{ppm}(67,3 \%)$ fue igual que con formalina a $10 \mathrm{ppm}(54,4 \%),\left(\right.$ Tukey, $\mathrm{Q}_{8,28}=$ $2,907, P>0,05)$.
El tamaño de las larvas en los tratamientos con formalina fue diferente entre los tratamientos y el control (2,30 mm) (Kruskal-Wallis, $\left.\mathrm{H}_{3,32}=28,141, P \leq 0,001\right)$, excepto el de $10 \mathrm{ppm}$ con 2,32 $\mathrm{mm}$ (Dunn, $\mathrm{Q}_{3,32}=0,663, P$ $>0,05)$. En los tratamientos con acriflavina hubo diferencias significativas (Kruskal-Wallis, $\mathrm{H}_{3,32}=11,623$, $P=0,009)$ por la concentración de 7,5 ppm que presentó larvas significativamente menores de 2,22 $\mathrm{mm}$ (Dunn, $\left.\mathrm{Q}_{3,32}=3,269, P<0,05\right)$. Con glutaraldehido también hubo diferencias significativas con el control $(2,28 \mathrm{~mm})$ (Kruskal-Wallis, $\mathrm{H}_{3,32}=3,010, P=0,045$ ), por la reducción con $200 \mathrm{ppm}$ (Dunn, $\mathrm{Q}_{3,32}=0,050, P<0,05$ ). Las diferencias entre los tratamientos fue significativa (ANDEVA, $\mathrm{F}_{8,80}=$ $35,11, P=0,0028)$. La longitud de larvas normales con formalina a $10 \mathrm{ppm}(2,31 \mathrm{~mm})$ fue igual que con acriflavina a 5 ppm $(2,26 \mathrm{~mm})$, (Tukey, $\left.\mathrm{Q}_{8,28}=2,97, P>0,05\right)$.

Otros estudios han reportado la disminución de los porcentajes de eclosión, supervivencia y larvas normales en determinados tratamientos (Grotmol et al. 2003, BenAtia et al. 2007, Katharios et al. 2007, Verner-Jeffreys et al. 2007, Stuart et al. 2010); sin embargo, hay pocos resultados sobre la longitud de las larvas (Stuart et al. 2010).

Cuando se utiliza agua tratada para lavar los huevos, usualmente los tratamientos no son mejores que los controles (Grotmol et al. 2003); sin embargo, en el presente estudio, los porcentajes de larvas normales con 2,5 ppm y $5 \mathrm{ppm}$ de acriflavina y las longitudes de las larvas con $10 \mathrm{ppm}$ de formalina fueron mejores que en los controles.

Rodríguez-Ibarra et al. (2011) con botete diana, Sphoeroides annulatus, reportaron buenos resultados con glutaraldehido $(50 \mathrm{ppm})$, pero no recomendaron usarlo por sus afectaciones. El tratamiento con glutaraldehido a $400 \mathrm{ppm}$ por $10 \mathrm{~min}$ es el tratamiento recomendado para desinfectar los huevos de varias especies de aguas frías, con periodos embrionarios prolongados (Salvesen \& Vadstein 1995); sin embargo, Katharios et al. (2007) no lograron reducir la carga bacteriana en huevos de espáridos del Mediterráneo a concentraciones sin efectos perjudiciales. Salvesen \& Vadstein (1995) reportaron afectación de la eclosión y supervivencia con este tratamiento.

En el presente estudio, los mejores resultados se lograron con acriflavina a $5 \mathrm{ppm}$ y formalina a $10 \mathrm{ppm}$, aunque los mejores porcentajes de supervivencia y de larvas vivas se lograron con acriflavina. El costo estimado para tratar un millón de huevos con acriflavina 5 ppm 
también fue menor (US\$ 0,15) que con formalina $10 \mathrm{ppm}$ (US\$ 0,67). El uso de la acriflavina está bien establecido (Elliott \& Amend 1978) y se utiliza en los centros de producción de juveniles, como agente profiláctico y terapéutico (Tiensongrusmee et al. 1989, Mohamed et al. 2000). El uso de formalina está aprobado en EE.UU. y se aplica en general (Holt 2005); sin embargo, Verner-Jeffreys et al. (2007) recomendaron no usarlo de existir otro tratamiento disponible, para evitar riesgos al personal. Por lo anterior, se recomienda el tratamiento con acriflavina a 5 ppm.

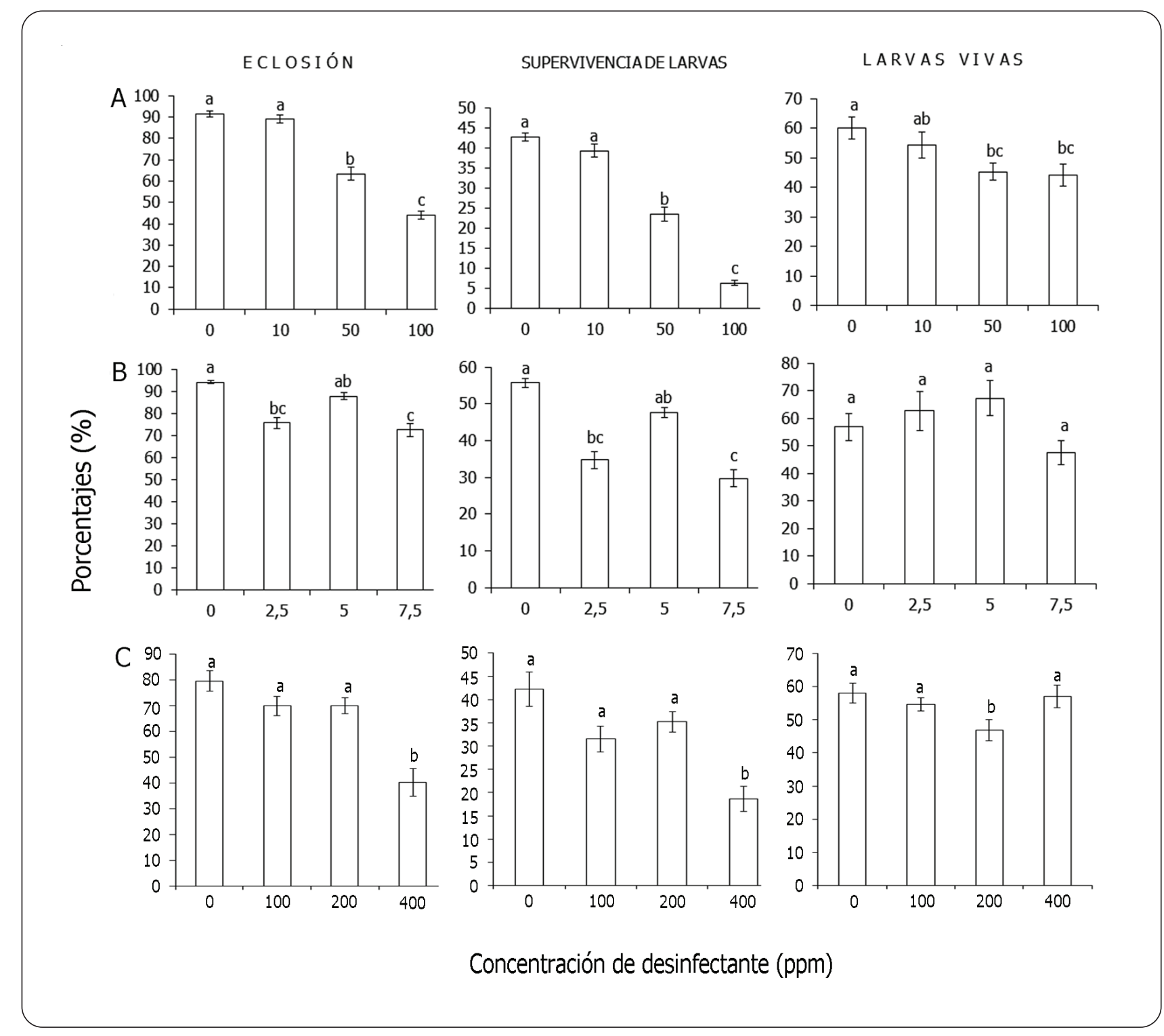

Figura 2. Porcentajes de eclosión, de supervivencia a la eclosión y de larvas normales, con los tratamientos profilácticos: A) formalina, B) acriflavina y C) glutaraldehido. Cada barra representa el promedio \pm error estándar de la media de las tres réplicas con las tres repeticiones $(n=9)$ en cada tratamiento aplicado a huevos del pargo flamenco Lutjanus guttatus. Las letras minúsculas diferentes indican diferencias significativas (ANDEVA, $P<0,05$ ) respecto a los controles / Hatching, survival and normal larvae percentages with prophylactic treatments with: A) formalin, B) acriflavine and C) glutaraldehyde. Each bar represents the mean \pm standard error of the mean of three replica repeated three times $(n=9)$ in each experiment with spotted red snapper Lutjanus guttatus, eggs. Different lower case letters indicate significant differences compared with controls (ANOVA, $P<0.05$ ) 


\section{Agradecimientos}

Los autores desean agradecer a los colegas del Laboratorio de Reproducción del CIAD por su cooperación y comentarios útiles sobre el manuscrito. La investigación fue financiada por los Proyectos 6299-K y 6299-A de la Comisión Nacional de Acuicultura y Pesca (CONAPESCA).

\section{LITERATURA CITADA}

Avilés-Quevedo A, JM Mazón-Suástegui \& F CastellóOrvay. 2008. Avances en el cultivo del pargo flamenco, Lutjanus guttatus, un ejemplo a seguir de los pescadores de Bahía Concepción, en Baja California Sur. Industria Acuícola 4: 4-7.

Ben-Atia I, S Lutzky, Y Barr, K Gamsiz, Z Shtupler, A Tandler \& W Kove. 2007. Improve performance of gilthead sea bream, Sparus aurata, larvae after ozone disinfection of the eggs. Aquaculture Research 38: 166-173.

Dehasque M, L Verdonck, P Sorgeloos, J Swings, P Léger \& K Kersters. 1991. Determination of the bacterial contamination in live food production systems in marine fish hatcheries in Southern Europe. In: Lavens P, P Sorgeloos, E Jaspers \& F Ollevier (eds). Larvi'91: Fish \& Crustacean Larviculture Symposium, pp. 399-402. Belgium, European Aquaculture Society Special Publication 15, Gent.

Douillet PA \& GJ Holt. 1994. Surface disinfection of red drum (Sciaenops ocellatus Linnaeus) eggs leading to bacteriafree larvae. Journal of Experimental Biology and Ecology 179: 253-266.

Elliott DG \& DF Amend. 1978. Efficacy of certain disinfectants against infectious pancreatic necrosis virus. Journal of Fish Biology 12: 277-286.

Gieseker CM, SG Serfling \& R Reimschuessel. 2006. Formalin treatment to reduce mortality associated with Saprolegnia parasitica in rainbow trout, Oncorhynchus mykiss. Aquaculture 253: 120-129.

Grotmol S, E Dahl-Paulsen \& GK Totland. 2003. Hatchability of eggs from Atlantic cod, turbot and Atlantic halibut after disinfection with ozonated seawater. Aquaculture 221: 245-254.

Hansen TK \& IB Falk-Petersen. 2001. Effects of egg disinfection and incubation temperature on early life stages of spotted wolfish. Aquaculture International 9: 333-344.
Holt GJ. 2005. Red drum aquaculture. American Fisheries Society Symposium 46: 457-463.

Ibarra-Castro, L \& L Alvarez-Lajonchère. 2011. GnRHa induced multiple spawns and volition spawning of captive spotted rose snapper, Lutjanus guttatus, at Mazatlan, Mexico. Journal of the World Aquaculture Society 42: 564574.

Katharios P, A Agathaggelou, S Paraskevopoulos \& CC Mylonas. 2007. Comparison of iodine and glutaraldehyde as surface disinfectants for red porgy (Pagrus pagrus) and white sea bream (Diplodus sargus sargus) eggs. Aquaculture Research 38: 527-536.

Mohamed S, G. Nagaraj, FHC Chua \& YG Wang. 2000. The use of chemicals in aquaculture in Malaysia and Singapore. In: Arthur JR, CR Lavilla-Pitogo \& RP Subasinghe (eds). Use of chemicals in aquaculture in Asia. Proceedings of the meeting on the use of chemicals in aquaculture in Asia 20-22 May 1996, Tigbauan, Iloilo, pp. $127-140$.

Moretti A, M Pedini Fernandez-Criado, G Cittolin \& R Guidastri. 1999. Manual on hatchery production of seabass and gilthead seabream 1: 1-194. FAO, Rome.

Rodríguez-Ibarra LE, MI Abdo-de la Parra, GA RodríguezMontes de Oca, CY Padilla-Aguiar, VY ZepedaMercado, G Velasco-Blanco \& N García-Aguilar. 2011. Efecto de la acriflavina, formalina y glutaraldehído sobre la desinfección y la eclosión de los huevos del botete diana Sphoeroides annulatus. Revista de Biología Marina y Oceanografía 46: 59-65.

Salvesen I \& O Vadstein. 1995. Surface disinfection of eggs from marine fish: evaluation of four chemicals. Aquaculture International 3: 155-171.

Stuart KR, M Keller \& M Drawbridge. 2010. Efficacy of formalin and povidone-iodine disinfection techniques on the eggs of three marine finfish species. Aquaculture Research 41: 838-843

Tiensongrusmee B, S Budileksono, S Cjhanstarasri, SKY Yuwono \& H Santoso. 1989. Propagation of seabass, Lates calcarifer in captivity. Seafarming Development Project, UNDP/FAO/INS/81/008/MANUAL/15, pp. 1-55.

Verner-Jeffreys DW, I Nakamura \& RJ Shields. 2007. Surface disinfection of Pacific threadfin, Polydactylus sexfilis, and amberjack, Seriola rivoliana, eggs. Aquaculture Research 38: 605-612.

Recibido el 18 de octubre de 2011 y aceptado el 26 de enero de 2012 